\title{
FACTORES DE RIESGO DE ANEMIA FERROPÉNICA EN NIÑOS Y ADOLESCENTES ESCOLARES DE LA CIUDAD DE TACNA
}

Responsable: Blgo. Guillermo Bornaz Acosta Miembro: Blgo. Soledad A. Bornás Acosta Miembro: CD. Milagros C. Bornaz Arenas Miembro: Dr. Luis Coronel Amorin

\section{RESUMEN}

El presente trabajo tuvo como objetivo determinar los factores de riesgo para anemia por deficiencia de hierro en niños y adolescentes escolares de 6 a 17 años de edad de la ciudad de Tacna.

Se realizó un estudio transversal en 247 escolares del distrito Gregorio Albarracin (cono sur) durante el año académico 2005. Se recolectaron datos demográficos, socioeconómicos, condiciones domiciliarias, ambientales y de ingesta de alimentos ricos en hierro. Se determinaron los niveles de hemoglobina y se analizaron muestras de heces en busca de infección de parásitos. Se llevó a cabo una discriminación estadística de los factores de riesgo.La prevalencia global de anemia fue de $38,6 \%$, de los cuales $9 \%$ sufrían de anemia grave. Las infecciones parasitarias tuvieron una incidencia de $47 \%$ y el $58,1 \%$ presentaron anemia ferropénica. Los resultados del análisis estadístico mostraron que la edad de 6 a 9 años, la renta familiar per cápita menor de $1 / 4$ de la remuneración mínima vital, la baja escolaridad del jefe de familia, las condiciones domiciliarias y ambientales inadecuadas y la ingestión de hierro biodisponible menor del $50 \%$ de la RDAestuvieron significativamente asociados a la anemia por deficiencia de hierro.

En conclusión, la anemia ferropénica en los escolares del cono sur de Tacna es un problema de salud pública en los grupos de mayor riesgo, y para contrarrestar su alta incidencia debe incrementarse el consumo de alimentos ricos en hierro, combatir y evitar las infecciones por parásitos y adoptar programas orientados a disminuir los factores de riesgo.

\section{ABSTRACT}

The following research had the objective to determine the risk factors for anemia by deficiency of iron in children and teenagers students between 6 and 17 years old in Tacna.

We made a transversal study in247 students from Gregorio Albarracin district, during the academic year 2005, recollected demographic datum, social and economic, living conditions, environment, and feeding nutriment rich in iron. We determined the levels of hemoglobin and analyze dregs samples looking for parasitizing infection then we analyze statistically the risk factors. The prevalence of global anemia was $38,6 \%, 9 \%$ suffered a grave disease. The parasitizing infection had an incidence of $47 \%$ and $58,71 \%$ presented ferropenic anemia. The result of the statistical test showed the big group between 6 and 9 years old. The family income less than $1 / 4$ of the minimum salary in Peru, the low academic prepare of the parents, house conditions and inappropriate environment, and the less feeding nutriment rich in iron, less than the $50 \%$ of the RDA was significantly associate to the deficiency of iron.

In conclusion, The Forropenic anemia in students from Cono Sur Tacna is a public health problem in the big risk group, to fight again the high incidence must increase the nutrition with food rich in iron, and avoid the parasitizing infection, adopting oriented programs to reduce the risk factors.

\section{INTRODUCCIÓN}

La anemia por deficiencia de hierro es uno de los problemas nutricionales de mayor magnitud en el mundo, es un problema frecuente de salud pública, tanto en países industrializados como en países en desarrollo, donde más de un tercio de la población mundial se estima que presentaron anemia en el año de 1999, número que corresponde a un tercio de la población mundial (1). Según algunos cálculos, en el año 2000 , la deficiencia de hierro y la anemia ferropénica afectaron a más de 3500 millones de seres humanos (1 y 2).
Los grupos más afectados por la anemia ferropénica en países industrializados son las embarazadas (18\%) y los preescolares $(17 \%)$, similar situación se dio en los países en desarrollo (mujeres embarazadas $56 \%$, escolares $53 \%$ y preescolares $42 \%$ ). Esta situación puede verse agravada por la presencia de infestaciones parasitarias, enfermedades infecciosas frecuentes y otras deficiencias alimentarias que pueden afectar directamente a la formación de la hemoglobina y/o a las pérdidas de sangre que ocasionan (3). En América Latina el número de niños anémicos en la década de los 80 fue de 13,7 millones, lo que equivale a una 
prevalencia de $26 \%$ (4). Un informe de la Organización Panamericana de la Salud (OPS), basado en estudios locales o estatales, señaló a Perú como el país con la mayor prevalencia de anemia en toda América Latina y el Caribe (57\%) seguido de Brasil donde $35 \%$ de los niños de 1 a 4 años sufrian de anemia (5). La importancia de las anemias nutricionales y de la deficiencia de hierro radica no solamente en su alta frecuencia sino en los trastornos funcionales que ocasionaron en ambos sexos, aun en su forma moderada (6). Numerosos estudios han mostrado que la anemia por deficiencia de hierro incrementa la morbilidad y la mortalidad, retrasa el crecimiento de los niños y dificulta la función cognoscitiva y el desarrollo escolar (7). En los adultos disminuye la capacidad de trabajo y dificulta la labor obstétrica (8). La corrección de la anemia mediante el tratamiento con hierro no conduce a mejores resultados en las pruebas mentales (5 y 9 ). Esto sugiere que si la anemia se presenta en el periodo crítico de crecimiento y diferenciación cerebral, observada en los niños menores de dos años, el daño puede ser irreversible.

La anemia por deficiencia de hierro resulta de la combinación de múltiples factores etiológico, Entre las causas inmediatas de esta carencia destacan la baja ingesta de alimentos fuentes de hierro, las pérdidas de este micronutriente por infecciones parasitarias y una baja absorción de hierro por ausencia de factores que la potencializan (carnes, ácidos orgánicos y otros) y/o presencia de inhibidores de su absorción ( $\mathrm{Ca}$, te, café, etc).

Cada vez más se reconoce que los niños en edad escolar, especialmente en los países en desarrollo, sufren de problemas de salud que pueden limitar su capacidad cognitiva (10). Por otro lado, el ambiente escolar ofrece un contexto adecuado para efectuar programas de salud pública de diferentes tipos, tales como los que se basan en la educación en salud, en suplementar los alimentos con hierro o en la prevención de infecciones parasitarias (11).

Es verdaderamente triste que en la mayoria de los paises latinoamericanos haya elevadas prevalencias de anemia por deficiencia de hierro moderada y grave, sobre todo si se toma en cuenta que se trata de trastornos prevenibles en la actualidad. Debe considerarse una tragedia que muchos millones de niños latinoamericanos hayan podido sufrir daño cerebral a edad temprana, con un retraso del desarrollo psicomotor que pudiera ser irreversible, lo que pudo evitarse, como resultados de las políticas de salud y nutrición en los diferentes países de la región. En el Perú son muy escasos los trabajos de prevalencia de anemia por deficiencia de hierro en niños y adolescentes escolares, especialmente en la región de Tacna; asi mismo, no hay investigaciones sobre los factores de
Riesgo que los predisponen a sufrir esta manifestación carencial.

Motivado por todos estos antecedentes, el objetivo de este trabajo de investigación fue determinar la prevalencia de anemia ferropénica y discriminar los posibles factores de riesgo en escolares y adolescentes matriculados en la jornada diurna de colegios del cono sur de la ciudad de Tacna durante el año académico de 2005, explorando la asociación entre la edad, sexo, nivel socioeconómico, saneamiento ambiental, parasitismo, tipo de dieta, con anemia ferropénica.

\section{MATERIALY MÉTODOS}

El estudio se realizó durante el año académico 2005 en los colegios de primaria y secundaria del distrito Gregorio Albarracín de la ciudad de Tacna. Se seleccionó una muestra de los escolares matriculados durante el año académico 2005. Sobre la base de un nivel de significación de $0,05 \%$ y un error de muestreo de 0,03 se calculo una muestra de 247 escolares de 6 a 17 años de edad. De cada centro educativo se escogieron escolares en forma aleatoria simple hasta completar la muestra calculada, Se excluyeron a los escolares que presentaron algún proceso patológico.

\section{Datos Demográficos:}

A cada escolar se le identificó con su nombre, fecha de nacimiento, sexo, edad, el nombre de la escuela o colegio. Todos los niños $y$ adolescentes registrados tuvieron la aceptación de sus padres, salvo 9 que no participaron en el estudio por pedido de sus padres.

\section{Factores socioeconómicos}

Se obtuvieron datos del padre o jefe de familia, su edad, sexo, tipo de trabajo, nivel educativo alcanzado y el ingreso económico familiar. Per cápita familiar. Se establecierón tres categorías: ingreso per cápita: menor que $1 / 4$ de la remuneración mínima vital (450 soles), ingreso entre $1 / 4$ a $1 / 2$ de la remuneración mínima vital y un ingreso mayor que $1 / 2$ de la remuneración mínima vital. Se determinarón tres categorias para la escolaridad del jefe de familia: analfabeto, estudios de primaria y estudios de secundaria a superior.

\section{Saneamiento Ambiental}

Aplicando un cuestionario, se obtuvo datos de cada escolar, de las caracteristicas de su casa- habitación, la disponibilidad de agua 
potable y tipo de instalaciones sanitarias. Se diseñó una tabla sobre 10 puntos de base a las condiciones domiciliarias y de saneamiento ambiental; a las familias que superaron los 5 puntos se les consideró con condiciones de saneamiento ambiental adecuadas; $y$ a las que no, con condiciones inadecuadas.

\section{Muestras de sangre}

Se extrajeron $5 \mathrm{ml}$ de sangre con anticoagulante y se determinó la concentración de hemoglobina $(\mathrm{Hb})$. La determinación de hemoglobina se llevó a cabo mediante el método de la cianometahemoglobina, utilizando un espectrofotómetro KarlZeiss.

\section{Clasificación de anemia}

Para clasificar los casos de anemia por deficiencia de hierro, se utilizaron los puntos de corte establecidos por la FAO y la OMS.

\section{Análisis de heces}

De cada individuo de la muestra constituida por 247 estudiantes, fueron colectadas en vasitos descartables dos muestras de heces en momentos diferentes, las cuales se sometieron a análisis en solución salina lugol, utilizando el método formol-éter o de Ritchie (11).

\section{Evaluación dietética}

El consumo de hierro y calorías se evaluó mediante un cuestionario recordatorio de 24 horas de consumo de alimentos ricos en hierro durante una semana, para establecer la frecuencia de consumo de calorías y alimentos ricos en hierro biodisponible. Para evaluar la asociación entre la frecuencia de consumo y la anemia se establecieron dos categorias: 1) ingestión inadecuada, menor o igual al $50 \%$ a menor del $100 \%$ del IDR; 2 ) adecuada, igual o mayor del $100 \%$ de IDR según el grupo de edad y sexo.

\section{Análisis de datos}

Todos los datos se analizaron con el programa estadístico Epi Info 6 para describir las variables dependientes e independientes. Se utilizo la prueba de chi cuadrado con un nivel de significación de $5 \%$ para establecer las asociaciones estadisticas entre las variables dependientes y las independientes. El grado de asociación entre anemia y sus posibles determinantes fue estimado por el cálculo de razón de cambios OLDS RATIO (OR) con un intervalo de confianza del $95 \%$.

\section{RESULTADOS}

Los valores promedio de hemoglobina de la muestra estudiada fue de 11,62 gramos de hemoglobina/dl, La prevalencia global de anemia fue de $3,87 \%$, donde el $9,6 \%$ presentó anemia grave $(>9,5 \mathrm{~g} / \mathrm{dl})$, el resto de la muestra entre anemia moderada y anemia leve.

En el Cuadro 1 se observan los valores promedio de hemoglobina según sexo y edad, presentando una reducción de prevalencia de anemia con el incremento de la edad $(p<0,05)$; no hubo diferencias significativas en la prevalencia de anemia entre el sexo masculino yel femenino.

CUADRO 1: Valores Promedio de Hemoglobina y sus Desviaciones Estándar (de) y Prevalencia de Anemia Según Sexo y Edad en Escolares de 6 a 17 Años del Cono Sur de la Ciudad de Tacna, 2003.

\begin{tabular}{|c|c|c|c|c|c|c|c|c|c|}
\hline \multirow[t]{2}{*}{ ENOD } & \multirow[t]{2}{*}{ N } & \multirow[t]{2}{*}{ x } & \multicolumn{2}{|c|}{ Hemoglebina } & \multicolumn{2}{|c|}{$\begin{array}{l}\text { PREALEROA } \\
\text { OEANEYYA }\end{array}$} & \multicolumn{2}{|c|}{ SH AVEMIA } & \multirow[t]{2}{*}{$p$} \\
\hline & & & Proness & of & P & 8 & l & 4 & \\
\hline $6-9$ & 128 & 4130 & 11,15 & 1348 & 13. & 216 & 9 & 5,4 & \\
\hline $10-14$ & 107 & 432 & 11,81 & 1,85 & 41 & 328 & 60 & 61,88 & $60 \%$ \\
\hline $15=17$ & 38 & $15 \%$ & $12 x$ & 1799 & 12 & 31,5 & $x$ & 004 & \\
\hline \multicolumn{10}{|l|}{590} \\
\hline mscaun & 134 & 518 & 115 & 100 & 52 & $x x$ & $\mathbb{Q}$ & 6120 & \\
\hline FaANo & tis & 5,75 & 11,73 & 1,07 & 4 & 1781 & 60 & 81,06 & \\
\hline TOTK & 277 & 1000 & $11: 2$ & 152 & 80 & 388 & 151 & $6,1\}$ & \\
\hline
\end{tabular}

La prevalencia de anemia disminuye con el incremento de la renta familiar per cápita $(p<$ $0,01)$,con el incremento de la escolaridad del jefe de familia $(p<0,01)$ con las condiciones ambientales adecuadas $(p<0,01)$ y la ingesta de hierro biodisponible, $(p<0,01)$, tal como se observan en el Cuadro 2. Además, en el mismo Cuadro se observan los resultados del total de las heces analizadas de los 247 escolares de la muestra, donde 118 individuos presentaron parásitos intestinales $(47,77 \%)$ de los cuales el $56,78 \%$ presentó anemia ferropénica $(p<0,01)$. Los niños y adolescentes no parasitados fueron $129(52,23 \%)$ y solo 29 presentaron anemia 
que representa el $11,74 \%$ de la muestra total y el $30,21 \%$ del total de los individuos que presentaron anemia.

CUADRO 2: Valores Promedios De Hemoglobina y sus Desviaciones Estándar (de) y Prevalencia de Anemia Según Renta Familiar, Escolaridad del Jefe de Familia, Condiciones Ambientales y Domiciliarias, Ingestión de Hierro Biodisponible y Análisis de Heces en Escolares de 6 a 17 Años del Cono Sur de la Ciudad de Tacna, 2005.

\begin{tabular}{|c|c|c|c|c|c|c|c|c|c|}
\hline \multirow{2}{*}{$\begin{array}{c}\text { REVTARAMUILAR } \\
\text { percapta }\end{array}$} & \multirow{2}{*}{$k^{\circ}$} & \multirow{2}{*}{ s } & \multicolumn{2}{|c|}{ Henogolotina } & \multicolumn{2}{|c|}{ Con Anemia } & \multicolumn{2}{|c|}{ SinAremia } & \multirow{2}{*}{$p$} \\
\hline & & & Promedio & DE & $r$ & \% & $n$ & $x$ & \\
\hline$\angle \mathrm{R} W \mathrm{~N}$ & 121 & 41,30 & 11,153 & 1.676 & 53 & 41,80 & 68 & 56,20 & \multirow{3}{*}{$<0,01$} \\
\hline MaYRRW & 78 & 4332 & 11,54 & 1256 & $x$ & 33,45 & 48 & 61,54 & \\
\hline$>Y_{2} R W$ & 48 & 1538 & 12288 & 1.074 & 13 & 27,08 & 35 & 7292 & \\
\hline \multicolumn{10}{|c|}{ ESCOLAROAD DEL JEFE OEFAVILA } \\
\hline ANGHBETO & 32 & 1286 & 1055 & 2,003 & 19 & 59,38 & 13 & 40,02 & \multirow{3}{*}{$<0,01$} \\
\hline PRMAQA & 112 & 4534 & 11,0 & 1,405 & 47 & 41,96 & 65 & 58,4 & \\
\hline secolodat & 103 & 41,70 & 1223 & 1,167 & 30 & 20,13 & 73 & 70.87 & \\
\hline \multicolumn{10}{|c|}{ CONDCCONES DOUCC LARES Y AVBIETRLES } \\
\hline NoECUNAS & 93 & 37,65 & 1120 & 1,748 & 42 & 45,16 & 51 & 5584 & \multirow[t]{2}{*}{ k001 } \\
\hline ADECUNDS & 154 & 62,35 & 11,88 & 1,306 & 54 & 36.06 & 100 & 64,35 & \\
\hline \multicolumn{10}{|c|}{ INGESTON DE HIERRO BIOOSPONBLE } \\
\hline$<5$ & 59 & 23,89 & 1100 & 1963 & 28 & 11,34 & 31 & 12,55 & \\
\hline $50-99$ & 5 & 38,86 & 11,71 & 1234 & 37 & 1488 & 59 & 23,88 & \\
\hline$>100$ & $x$ & 37,25 & 11,93 & 1,388 & 31 & 12,55 & 61 & 24,70 & 0001 \\
\hline \multicolumn{10}{|c|}{ ANLUSIS DE HECES } \\
\hline PRRASTADOS & 118 & $47, \pi$ & 11,10 & 1000 & 67 & 56,78 & 51 & 4322 & \multirow{3}{*}{$<0,01$} \\
\hline nopustices & 120 & 5223 & 1210 & 1,103 & 29 & 2,48 & 100 & 77,52 & \\
\hline TOTAL & 247 & 10000 & 11,62 & 1,52 & 96 & 38,87 & 151 & 61,13 & \\
\hline
\end{tabular}

Con el análisis estadistico de razón de cambio (OR), se identificó que la renta familiar per cápita inferior a $1 / 4$ del salario mínimo vital (SMV), la escolaridad del jefe de familia analfabeta y las condiciones ambientales inadecuadas, el parasitismo intestinal y la ingestión de hierro biodisponible menor al $50 \%$ a menor del $99 \%$ de la IDR presentaron una asociación significativa con la anemia $(p<0.05)$ tal como se observa en el Cuadro 4. No hubo asociación del grupo de escolares de sexo masculino con el grupo de sexo femenino con la anemia con el análisis. Con relación a la edad, se ajusto el análisis de este factor con las otras variables, verificándose que los niños de edad entre 6 a 9 años presentaron un riesgo mayor de desarrollo de anemia, según el grupo de 10 a 14 años (Cuadro $N^{\circ} 3$ ).
CUADRO 3: Asociación entre la Anemia y los Factores Socioeconómicos Ambientales, Demográficos, Ingesta de Calorías y de Hierro Biodisponible en Niños y Adolescentes Escolares de 6 a 17 Años, del Cono Sur de la Ciudad de Tacna, 2005.

\begin{tabular}{|c|c|c|}
\hline VARIABLE & $\begin{array}{l}\text { ORAJUSTADO } \\
\text { (IC 95\%) }\end{array}$ & p \\
\hline \multicolumn{3}{|c|}{ RENTA FAMILIAR per cápita } \\
\hline$>1 / 4$ RMV & $1,6(1,2 \mathrm{a} 2,3)$ & $<0,01$ \\
\hline$<1 / 4 a^{1} / 2$ RMV & $1,2(1,0 \mathrm{a} 1,9)$ & 0,09 \\
\hline$\angle 1 / 2 R M B$ & 1,0 & \\
\hline \multicolumn{3}{|c|}{ ESCOLARIDAD DEL JEFE DE FAMILLAA } \\
\hline Analffabeto & $1,5(1,0 \mathrm{a} 2,1)$ & $<0,05$ \\
\hline Primaria & $1,2(1,0 \mathrm{a} 1,9)$ & 0,12 \\
\hline Secundaria a más & 1,0 & \\
\hline \multicolumn{3}{|c|}{ CONDICIONES DOMICILIARIAS Y AMBIENTALES } \\
\hline Inadecuadas & $1,4(1,0 \mathrm{a} 1,6)$ & $<0,05$ \\
\hline Adecuadas & 1,0 & \\
\hline \multicolumn{3}{|l|}{ EDAD } \\
\hline 6 a 9 años & $1,6(1,0 \mathrm{a} 2,1)$ & $<0,05$ \\
\hline 10 a 14 años & $1,2(0,8 a 1,6)$ & 0,30 \\
\hline 15 a 17 años & 1,0 & \\
\hline \multicolumn{3}{|c|}{ INGESTA DE HIERRO BIODISPONIBLE } \\
\hline$<50$ & $1,6(1,0,2,1)$ & $<0,01$ \\
\hline 50 a 99 & $1,5(0,9 a 1,9)$ & $<0,05$ \\
\hline$>100$ & 1,0 & \\
\hline
\end{tabular}

\section{DISCUSIÓN}

En el presente estudio, las prevalencias encontradas en niños y adolescentes del distrito de Gregorio Albarracin $(38,9 \%)$, comparadas con otros estudios, fueron superiores a las de Argentina $(18 \%)$, Chile $(2,1 \%)$, Cuba $(12,9 \%)$, Colombia (6\%), Ecuador (16,6\%), Brasil (Bahia, 28,9 y $32,2 \%$; Sergipe, $27,6 \%$; Arcaju, $26,6 \%$ ), Lima $(9,9 \%)$; similares a los encontrados en adolescentes en Brasil (Parna, 37,7\%) e inferiores a las encontradas en Brasil (Pernambuco, 43\%; Sao Paulo, 51\%), ACC/SCN $(46 \%)$, Tanzania $(66,8$ y $43 \%)$ y Zanzibar $(62$ y $57,6 \%)$.

- El objetivo principal en el presente trabajo fue discriminar la asociación entre las variables que probablemente determinan la presencia de anemia ferropénica en niños y adolescentes escolares del distrito Gregorio Albarracín de la ciudad de Tacna. Se postuló que la ocurrencia de anemia está asociada a causas estructurales y básicas, representadas por las condiciones 
socioeconómicas de las familias de los escolares, el consumo de alimentos, la exposición a infecciones parasitarias.

En este estudio se encontró que una frecuencia de parasitismo relativamente alta $(48,9 \%)$ comparadas con los resultados encontrados de Brasil, Ecuador y Colombia (13) determina que la parasitosis intestinal debe ser considerada un factor de riesgo para anemia asociada al inadecuado consumo de alimento y saneamiento ambiental, porque exacerba las pérdidas de hierro generando hemorragias $y / o$ inhibiendo su absorción a nivel intestinal.

La renta familiar per cápita inferior a una cuarta parte del salario mínimo vital estuvo asociada a la anemia que presenta esta población en estudio, resultado que está en concordancia con hallazgos de otros investigadores de Latinoamérica $(14,15)$. Resalta que cerca del $50 \%$ de los niños y adolescentes que participaron en este estudio pertenecían a familias que tenían una renta familiar per cápita inferior a un cuarto de la remuneración mínima vital, situación que estaría influyendo sobre las condiciones sociales, ambientales, ingesta de calorías y hierro biodisponible, que se confirma con los resultados del Cuadro de las condiciones ambientales y domiciliarias, donde se observa que el $37.7 \%$ presentaron condiciones inadecuadas. El ingreso económico puede influir sobre la ocurrencia de anemia de diversas maneras, en especial sobre las posibilidades de adquirir y utilizar servicios higiénicos y otros servicios esenciales para el mantenimiento y cuidado del estado de salud. Sin embargo, los individuos de estratos socioeconómicos más privilegiados también presentaron una prevalencia relativamente alta de anemia. Estos resultados podrían ser atribuidos a factores culturales que definen los hábitos nutricionales independientemente del nivel socioeconómico.

Por otro lado, nuestros resultados mostraron que las condiciones ambientales y domiciliares inadecuadas pueden influir sobre la ocurrencia de anemia, posiblemente favoreciendo que los escolares estén más expuestos a procesos infecciosos y parasitosis; por lo que debe ser considerado como factor de riesgo, dado que la asociación es similar a la que obtuvo con la renta familiar percápita.

Los jefes de familia analfabetos fueron solamente 32 , que representaron el $12,9 \%$ de la muestra, cifra mucho menor que la encontrada en un estudio de Bahía-Brasil (1); sin embargo, la prevalencia de anemia fue elevada $(59,38 \%)$, demostrando una asociación entre la baja escolaridad con la anemia; probablemente este factor esté afectando la ingesta de alimentos y las condiciones ambientales.

- Los niños cuyas edades oscilan entre 7 y 9 años de edad constituyen el grupo más vulnerable a sufrir anemia ferropénica. Es interesante indicar la relación inversa que existe entre la prevalencia de anemia ferropénica y la edad, conforme la edad se incrementa el porcentaje de anémicos disminuye, situación que se ha encontrado también en otros estudios. FUJIMORI, BRITO, TATALA y colaboradores observaron que la anemia ferropénica fue más frecuente en escolares menores de los 12 años. Probablemente debido a una menor ingesta y mayores necesidades de hierro biodisponible $y$, además, a una mayor exposición a infecciones parasitarias.

- Los niños y escolares adolescentes, tanto mujeres como varones de la muestra en estudio, no presentaron diferencias significativas de prevalencia de anemia, tal como otras investigaciones anteriores $(15,16)$.

- Por todo lo expuesto, se recomienda implementar un plan estratégico de salud orientado a disminuir los factores de riesgo que generan este estado carencial.

\section{CONCLUSIONES}

1. En los escolares del cono sur de la ciudad de Tacna existe una alta prevalencia de anemia ferropénica, lo que constituye un problema de salud pública.

2. La edad de 6 a 12 años, renta per capita inferior a $1 / 4$ de la remuneración mínima vital, las condiciones domiciliarias y ambientales inadecuadas, el analfabetismo, el parasitismo intestinal y la ingesta de hierro biodisponible influyeron significativamente sobre la presencia de este estado carencial.

3. Los factores de riesgo encontrados en este estudio fueron: la edad, la renta familiar per capita, la escolaridad del jefe de familia, las condiciones domiciliarias y ambientales, y las infecciones por parásitos.

\section{BIBLIOGRAFÍA}

1. Brito L., Barreto M., Silva R., Asís A.m., Reis M.g., Parraga I. Y Blanton R.e.: Factores de Riesgo para anemia por deficiencia de ferropénica en crianzas e adolescentes parasitados por helmintos intestinales. Pan Am J 
Public Health 2003, 14(6): 422-430.

2. United Nations Children's Fund, United Nations University, World Health Organization, Iron deficiency anaemia assessment, prevention and control. A guide for programmer managers. Geneva: WHO; 123. 2001.

3. Vásquez Garibay EM."Anemia en la infancia". Rev Pan Salud Pública 2003; 113(6): 2-5.

4. Horwitz A. "El costo de la malnutrición". En: Organización Panamericana de la Salud, eds. Vigilancia Alimentaria y Nutricional en las Américas. Washington, DC: Organización Panamericana de la Salud; 1989. Pp 19-54(516).

5. Mora JO y Mora LM. Deficiencias de micro nutrientes en América Latina y el Caribe: Anemia ferropriva. Washington, DC: Organización Panamericana de la Salud; 1997.

6. Quizhpe E., San Sebastian M., Hurtig A.K. y Llamas A.: Prevalencia de anemia en escolares de la zona amazónica de Ecuador. Rev Pan Salud Pública 2003, 13(6): 355-360.

7. Tatala s., Svanberg V. and Madura B.: Low dietary iron availability is a major cause of anemia: a nutrition survey en the Lindi District of Tanzania. Am J Clin Mutr 1998; 68: 161-178.

8. Pollit E.: Early iron deficiency anemia and later mental retardation. Am J Clin Nutr 1999, 69(1):45.

9. De Andraca I, Castillo M, Walter T. Psychomotor develop and behavior and iron deficiency infants. Nutr Rev 1997; 55: 125-32.

10. Stenberg RJ, Grigorenko L, Nokes C. Effects of childen's ill health on cognitive development. En young ME, ed. Early child development. Washington, DC.World Bank, 1997. Pp 85-125.

11. Botero D, Restrepo M. Parasitosis humanas. $3^{a}$ ed. Medellín: Corporación para investigaciones Biológicas (CIB); 1998.

12. Agudelo GM, Cardona OL, Posada M, Montoya MN, Ocampo NE, Marin CM, Correa MC y Lopez C. Prevalencia de anemia ferropénica en escolares y adolescentes, Medellin, Colombia. Rev Panam Salud Publica 2003; 13(6):376-85.

13. Organización Mundial de la Salud. Anemias nutricionales. Ginebra OMS; 1970 (Estudio Básico $\mathrm{N}^{\circ} 24$ ).

14. Fujimori E, Szarfarc SC, Oliveira IMV. Prevalencia de anemia e deficiencia de ferro en adolescentes do sexo femenino: Toboao da Serra, SP, Brasil. Rev Lat Am Enfermagen 1996; 4(3): 49-63.

15. Ichimura TT. Anemia e desnutricao en escolares ingressantes nas escolas estuduais de Maringa, PR (dissertacao), Sao Paulo; Facultade de Saude Publica. Departamento de Nutricio de Sao Paulo; 1994.

16. Tsuyuoka, R, Bailey JW, Guimaraes AMDM, Gurgel RQ, Cuevas LE. Anemia and intestinal parasitic infections in primary school students in Aracaju, Sergipe, Brazil. Cad Saude Pública 1999; 15(2): 413-21. 